\title{
Gold Leads to PRTs for Monitoring High Temperatures
}

\author{
$J R$ Greenwood, P R N Childs and P Chaloner* \\ Thermo-Fluid Mechanics Research Centre, School of Engineering, \\ *School of Chemistry, Physics and Environmental Sciences, \\ University of Sussex, Brighton, United Kingdom, BN1 9RH.
}

Received 12 May 1999; and after revision 27 August 1999

For some aerospace and automotive applications, it has been difficult to produce instrumentation for the harsh environments encountered. Problems include high temperatures, high centrifugal forces, corrosive exhaust gases, and potential damage from high velocity particulates. This paper reports the design and development of a thin film instrument for temperature monitoring. The device comprises two gold tracks for use as excitation leads, and a platinum resistance element, both applied to a chemically robust electrical insulator. The gold tracks were deposited using a commercial gold ink consisting of a solution of a gold mercapto balsamate complex in an organic solvent. Most of the organic solvent evaporates when the ink is applied, and the residual organic material is removed on fusion. Gold is the metal of choice because of its resistance to oxidation and the chemical and thermal stability of the films produced. Gold ink is particularly easy to apply, the process is readily controlled and no expensive equipment is required. This paper describes the application of the gold ink and the analysis of the films produced, along with a description of the sensor fabrication and performance.

Application of thin film temperature instrumentation to turbomachinery components has been reported by Jones (1). One possibility is the use of vitreous enamel or polyimide as a thermal and electrical insulating substrate. Alternative substrates include vacuum deposited alumina or silicon monoxide. It is also possible to deposit a thermal barrier coating, these only being restricted by the limitations of their thermal stability. These insulating substrates can then be painted with small tracks of platinum to form a resistive temperature transducer. Gold tracks are used to provide electrical excitation and monitor the change in resistance of the sensor. Both gold and platinum tracks have been deposited using 'metallo-organic' inks. (The term metallo-organic is that used commercially to describe this ink; there are in fact no metal-carbon bonds in the gold complex). Sensors can be produced capable of withstanding accelerations of over $30,000 \mathrm{~g}$. This instrumentation may be used for steady state or transient temperature and heat flux measurement. Heat flux is determined by measuring the rate of change of temperature in transient operating conditions.
Gold ink has been used to produce tracks for the excitation and output signals from platinum resistance thermometers (PRT). The purpose of these devices was to measure the temperature at various points within an automotive catalytic converter. The construction of the sensors required the application of gold tracks onto alumina rods $0.7 \mathrm{~mm}$ in diameter. The gold was then trimmed using an iodine/potassium iodide etchant. The resistance element was fabricated from platinum ink. For this application, the use of gold was beneficial, as its specific electrical resistance $\left(2.2 \times 10^{-8} \Omega \mathrm{m}\right)$ is lower than that of platinum $\left(10.6 \times 10^{-8} \Omega \mathrm{m}\right)$, which ensures the isolation of the voltage drop in the measurement. An additional advantage is that the electrical conductance of gold is comparable with that of copper $\left(1.7 \times 10^{-8} \Omega \mathrm{m}\right)$, but, unlike copper, gold is highly resistant to oxidation.

Metal-organic materials have been used in metal track deposition for integrated circuits, with line widths of $1 \mu \mathrm{m}$ being achieved. The metal compounds decompose on exposure to electron beam irradiation producing high-resolution tracks. This method is 
reported by Berry (2) who used alkyl gold and palladium fluorides, such as tetrakis isobutyl diaurum difluoride and dicyclohexyl dipalladium difluoride. It has been reported by Pai (3) that in some instances gold from 'metallo-organic' inks is comparable with material obtained by vacuum deposition. However, the platinum films prepared from inks were not similar to those from vacuum deposited platinum and showed some evidence of interlayer contamination. This manifested itself in some non-linearity of the I-V characteristic, indicative of a non-Ohmic conductor. The performance was improved by annealing at $800^{\circ} \mathrm{C}$.

The gold-based ink (M6064A Engelhard-CLAL) contains mainly a gold thiol derivative in an organic solvent, most of which evaporates when the ink is applied. The residual organic material is removed on fusion. The composition of the gold mercapto balsamate complex was provided by the manufacturer. Similar types of metal ink although known as 'metallo-organic inks' actually contain carboxylate salts of the metals, which are ionic and are described by both Vest (4) and Hendricks and McDonald (5) who used them to fabricate metalloorganic films. The term 'metallo-organic' is not strictly correct for this material either, as the compounds contain metal-sulfur rather than metal-carbon bonds.

Scanning electron microscopy and Rutherford backscattering spectroscopy were used to analyse the surface structure and composition of the fused gold film. An energy dispersive $\mathbf{x}$-ray spectrum was recorded after annealing, to give a semi-quantitative assessment of possible interlayer diffusion or ageing of the surface that could cause subsequent loss of measuring accuracy or stability.

In the following section we describe the deposition and annealing of the 'metallo-organic' gold ink. Following this, the results of the micro-structural analysis of the gold films through mass spectrometry, Rutherford backscattering and energy dispersive $\mathbf{x}$-ray spectroscopy are presented. A calibration of the sensor is provided in the last section, where we also consider the performance of the sensor.

\section{SENSOR MANUFACTURE}

The composition of the gold ink was provided by Engelhard-CLAL; it consists of mercapto balsamate complex (57\%), ethyl acetate (2-3\%), solvent naphtha (heavy petroleum) (5-10\%), terpineol (10-15\%), turpentine (15-20\%) and rhodium 2-ethyl hexanoate $(<0.5 \%)$. The mercapto balsamate complex contains a gold-sulfur linkage. The metal ink is a true solution, not a colloid. The gold complex decomposes during firing to form a continuous film. The more volatile solvents evaporate first and this is followed by the gradual thermal decomposition of the gold thiolate complex. The gold deposits on the substrate in a facecentred cubic structure (6).

The ink can be applied to the substrate by a variety of techniques ranging from thick-film screening, ink-jet printing, spin-on, spray, or dip methods to hand-brush painting, to produce the required film. The sample is then heated to $125^{\circ} \mathrm{C}$ to remove any low boiling solvent that did not evaporate during deposition and then to $760^{\circ} \mathrm{C}$ to decompose the metal complex to produce a film. The change in volume of the film from organic solution to inorganic film is significant, so in order to achieve the required thickness several applications and firings were made to give a multi-layer film. Each firing resulted in a layer of between 50 and $200 \mathrm{~nm}$ in thickness. Once the desired parameters of electrical resistance and thickness of the gold sensor film were achieved, the sample was subjected to further heat annealing treatment for 4 hours at $800^{\circ} \mathrm{C}$. In this study, ink was applied directly to an alumina rod of diameter 0.7 $\mathrm{mm}$. A process of layering was used to give an average thickness of $30 \mu \mathrm{m}$, in order to reduce the electrical resistance of each track to $1 \Omega$. To achieve this, seven applications and firings were necessary. The resistance track of platinum used for this sensor was Engelhard CLAL's $05 \mathrm{X}$ metallo-organic platinum ink, with $10 \%$ metal content, which was fused at $750^{\circ} \mathrm{C}$, with an average thickness of $200 \mathrm{~nm}$ per layer.

The gold ink was applied over the entire surface of the alumina rod, to optimize coverage. The required

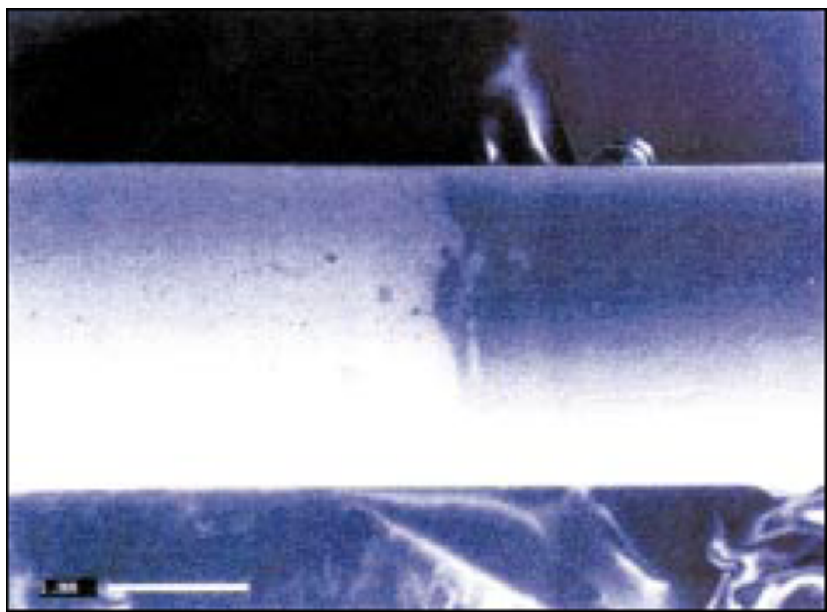

Figure 1 Scanning electron microscope image of the fused gold applied to an alumina rod. 
pattern was then etched using a standard photo-resist pen and an iodine/potassium iodide etchant (7). The result is shown in Figure 1.

\section{MICROSTRUCTURAL AND CHEMICAL ANALYSIS}

The structure of the gold layers applied to the alumina was studied using the ion beam analysis technique of Rutherford backscattering spectroscopy (RBS).

Figure 2 shows the experimental and theoretical backscattering spectrum derived from a model based on the tabulated scattering cross section and the stopping power to a depth of $2 \mu \mathrm{m}$ (3.0 MeV van der Graaf accelerator). Pai (3) used similar gold metallo-organic ink (8243) from Engelhard-CLAL and spun it onto silicon substrates. The films this produced gave rise to a similar shaped spectrum after annealing at $600^{\circ} \mathrm{C}$ for one hour.

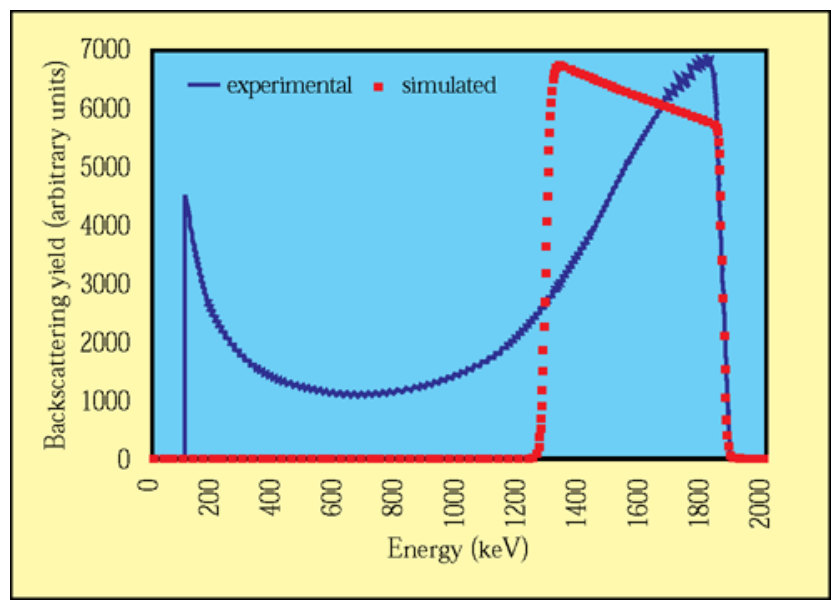

Figure 2 RBS energy spectrum of ${ }^{4}$ He backscattered from fused gold paint on an alumina substrate.

The experimental spectrum deviates from the simulated result because of the energy dependence of the scattering cross section and the uneven nature of the substrate surface. There is a possibility that interlayer diffusion might bring aluminium and oxygen into the penetration range, giving rise to small peaks hidden in the low energy region of the spectrum. This is due to the lower atomic weight of aluminium and oxygen compared with gold, and the uneven surface of the sample. A further uncertainty is caused by the multiple backscattering events that can also contribute to the unknown tail end area of the spectrum, at low energy. There was no definite evidence in the spectrum that the

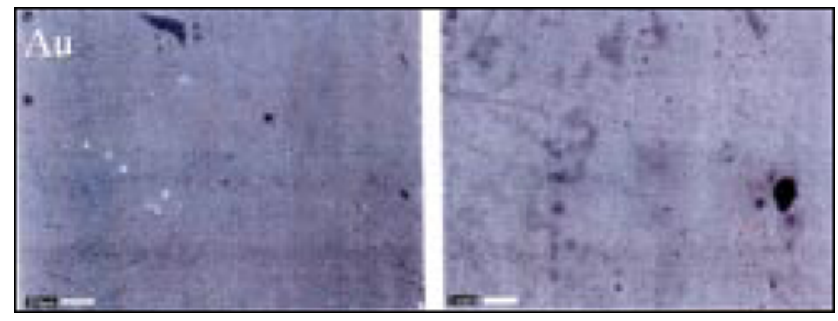

Figure 3 Scanning electron microscope image of the surface of fused gold.

elemental components of the alumina had diffused into the gold. Peaks were obtained in the spectrum at the energy level expected for elemental gold (channel 726, $1853 \mathrm{keV}$ ). It was beyond the scope of this investigation to ascertain whether there was any significant gold/alumina interlayer diffusion. In a relatively small gold surface irregularity, the energy loss of the particles traversing it is small, and therefore the signal has a sharp step at the energy edge of the element. For larger irregularities, a maximum develops and the decrease is more gradual. Apart from the sharp gold peak, the spectrum is characteristic of a thick sample. The substrate surface was rough, and the multiple gold layers were applied by hand, making the surface under investigation non-uniform. Chu (8) suggests that the sharp rise at the energy level corresponding to gold on the surface edge followed by the drop towards decreasing energies is indicative of lateral non-uniformity. This is corroborated by the fact that the surface of the specimen was irregular, as seen in the surface scan in Figure 3.

The gold and platinum both exhibit excellent adhesion to the substrate. Ultrasonic cleaning was unable to dislodge the deposited metal and there was no evidence of material removal as a result of practical adhesion tests.

An energy dispersive $x$-ray (EDX) spectrum was recorded using a Leo S420 scanning electron microscope to identify, semi-quantitatively, elements on the surface of the film. Figure 4 shows dominant gold peaks being identified through the collection of emitted energy, characteristic of elemental gold on the surface of the sensor.

\section{SENSOR PERFORMANCE AND CALIBRATION}

The calibration of the PRT was accomplished using a null-balanced Wheatstone bridge. The output from a resistance temperature device is a resistance change $(\Delta R / R)$, which can be observed using a Wheatstone 


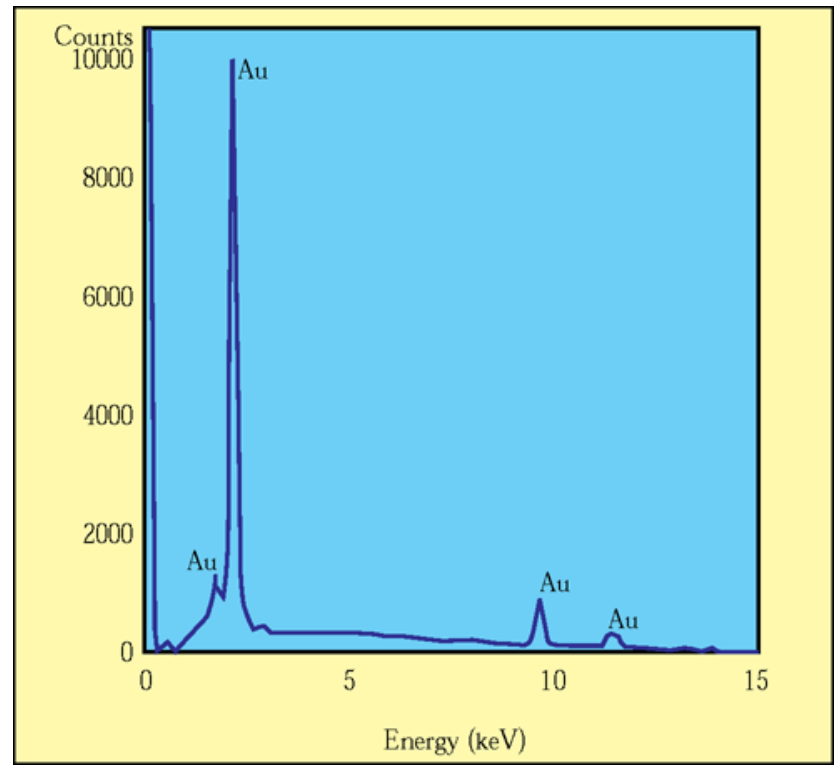

Figure 4 Energy dispersive $x$-ray $(E D X)$ spectrum of fused gold on an alumina substrate through a beryllium window at $20 \mathrm{keV}$.

bridge. Any resistance change, $\Delta R / R$, in the lead wires will produce an error in the readout. Reduction of these errors was achieved by using a three-wire-system, shown in Figure 5. Consequently, the temperature induced resistance change in the wires was cancelled. Three 80 $\mu \mathrm{m}$ diameter copper wires were soldered onto the gold lead tracks and then cemented in place with silica-based high-temperature ceramic cement. These small diameter wires were soldered to termination pads for connection to the data acquisition system. The gold lead tracks had a nominal resistance of $1 \Omega$ at room temperature before attachment of the $3 \Omega$ resistance wires.

The resistance versus temperature relationship was determined by heating the sensor and a DP251 precision digital reference RTD (calibrated to ITS-90 in compliance with ISO 10012-1 and ISO 9001),

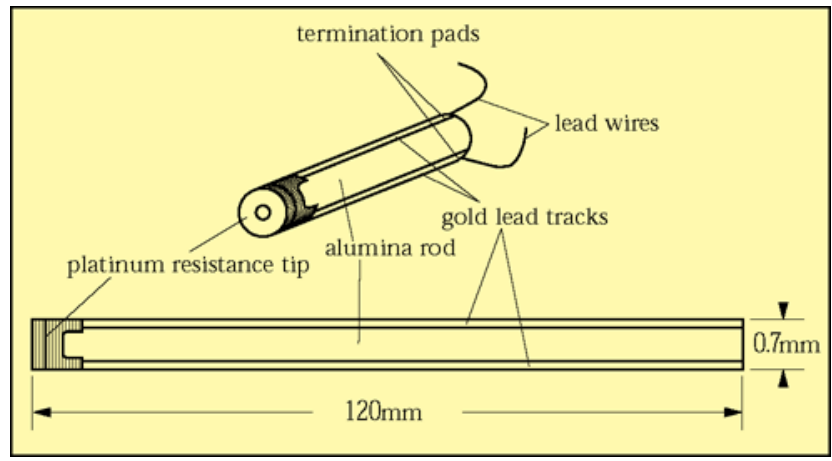

Figure 5 Schematic of the platinum resistance thermometer (PRT) sensor. within a heating coil. The resistance of the gauge over a set temperature range was then monitored. A low temperature reference point was established at the ice point.

The value of the fundamental coefficient $\gamma$, is a convenient measure of platinum purity. Most manufacturers use $\gamma=0.003920\left(\mathrm{~K}^{-1}\right)$ for a purity of platinum that gives a linear resistance - temperature relationship within $\pm 1.2 \%$ up to $1000^{\circ} \mathrm{C}$. For the platinum paint used for this PRT, the fundamental coefficient was $\gamma=0.001588\left(\mathrm{~K}^{-1}\right)$. This indicates the presence of impurities and is expected as the properties of bulk materials and thin films differ. The reader is referred to Chopra and Kaur (9) for a more comprehensive description of thin films and their applications.

The $\mathrm{R}^{2}$ variability of the recorded data was 0.993 . The characteristic equation from Figure 6 , was $\mathrm{y}=$ $0.6636 x+417.81$. The $\pm 3 \sigma$ limit for the temperature versus resistance characteristic equation was $\pm 1.06^{\circ} \mathrm{C}$. Inverting for the error in resistance of the characteristic, the $\pm 3 \sigma$ limit for the resistance versus temperature characteristic was $\pm 1.68 \Omega$.

The resistance ratio $\left(R / R_{0}\right)$, is the ratio of a resistance at any temperature to that at $0^{\circ} \mathrm{C}$. The fundamental interval of the PRT over the temperature range $0-100^{\circ} \mathrm{C}$, was $66 \Omega$. In commercial PRTs, the resistance at $0^{\circ} \mathrm{C}$ (according to DIN 43760:1980 and BS1904:1984) (10) is taken as $R_{0}=100 \Omega$. For the platinum resistance film used in this experiment, $R_{0}$ was greater than this $\left(R_{0}=418 \Omega\right)$, again, due to impurities. There are two advantages of a sensor with high resistance; the first is that the effect of any connection resistance within the circuit becomes negligible. Secondly, the relatively high voltage output produced by the device makes any thermally induced

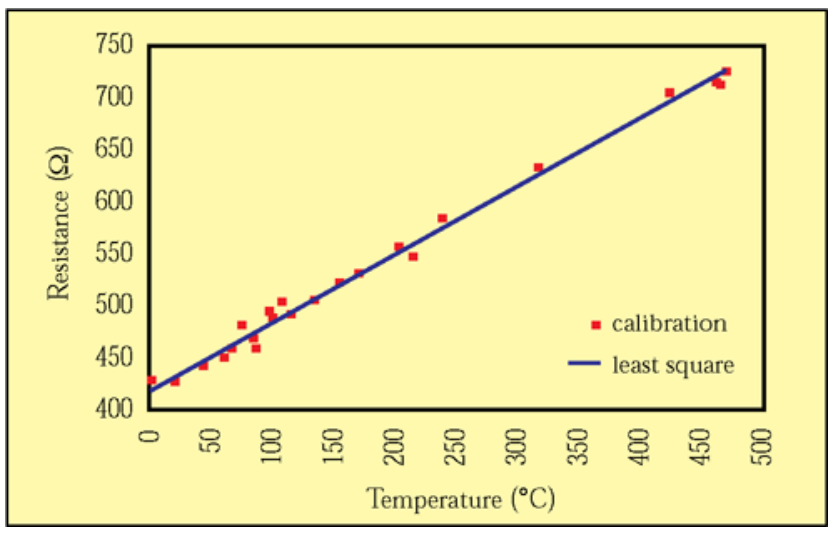

Figure 6 Platinum resistance thermometer (PRT) calibration characteristic.

x After annealing at $800^{\circ} \mathrm{C}$ for 4 hours - After annealing at $600^{\circ} \mathrm{C}$ for 1 hour 


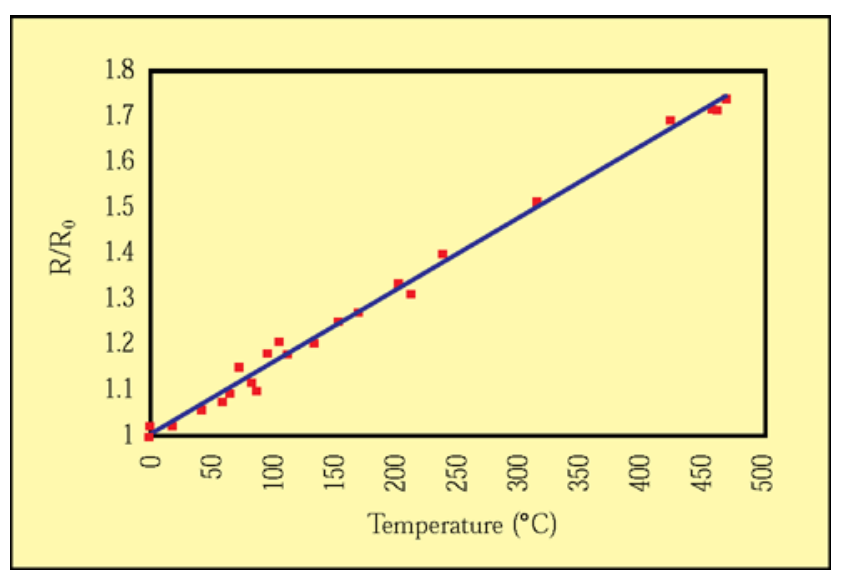

Figure 7 Sensitivity - defined as the slope of the temperature measuring function.

emf's, at intermediate junctions, also negligible. The sensitivity of the sensor over the calibrated range of temperatures was $0.0016^{\circ} \mathrm{C}^{-1}$ (Figure 7 ).

The base metal additions to the ink (2-3\%) are oxidized during firing (7). Either these impurities can form a glass, which acts as an interface bond between the substrate and the noble metal, or they may form a reaction product with the substrate to provide a chemical bond. In the multi-layered thin films used in this sensor, these impurities may also affect the electrical resistance. For example, Engelhard-CLAL reports that the resistance of the platinum paint is greater than 100 $\Omega$, which was confirmed. However, several layers of gold resinate were required to minimize the resistance of the lead tracks to a value of $\sim 1 \Omega$.

The calibration of the sensor was performed twice, once after annealing for one hour at $600^{\circ} \mathrm{C}$ and again after four hours at $800^{\circ} \mathrm{C}$. All measured points are shown (Figures 6 and 7 ), where each point represents a single measurement. The effects of annealing the sensor at the higher temperature reduced measurement errors. It may therefore be prudent to anneal the sensor for longer to maximize the interlayer diffusion between the gold layers making the tracks more homogenous. A further thin ceramic protective covering may be considered, as the purpose of this sensor is to measure the temperature of exhaust gases in a automotive engine, within the monolith of a catalytic converter. A coating of $\mathrm{Al}_{2} \mathrm{O}_{3}, \mathrm{SiO}$ or AlN could be sputtered onto the probe to protect the resistance track from mechanical and chemical incursion.

\section{CONCLUSIONS}

This study has demonstrated the feasibility of developing temperature measurement sensors for use in harsh environments. The ease of application of the metal complex inks, their robust nature after fusing, and compatibility with photolithographic processes makes them ideal tools for robust instrumentation without having to rely on expensive vacuum deposition techniques. SEM images have shown that although the films may have an irregular surface, there is no evidence of de-lamination or cracking. Results were obtained both at room temperature and after annealing at $800^{\circ} \mathrm{C}$ for four hours. The RBS and EDX spectra show the bulk material on the surface of the alumina as being gold with no evidence of contamination. The PRT sensor has been calibrated from 0 to $470^{\circ} \mathrm{C}$ giving a linear characteristic.

\section{ACKNOWLEDGEMENTS}

The support of the CASE studentship for $J$ R Greenwood by the EPSRC and Rolls-Royce plc is gratefully acknowledged. The authors also thank Dr D E Hole and Mr D P Randall for their assistance in performing the spectral and microscopic analysis presented in this article, $\mathrm{Mr} W \mathrm{~K}$ Ong for manufacturing the sensor, and $\mathrm{Mr} \mathrm{M}$ Parkhurst of Engelhard-CLAL (UK) Ltd for supply of information.

\section{ABOUT THE AUTHORS}

Peter Childs is a Senior Lecturer in the School of Engineering at the University of Sussex. He lectures mechanical design, fluid mechanics and heat transfer. He has interests in air breathing devices ranging from gas turbine and internal combustion engines to medical ventilators. In 1999 he was the winner of the American Society of Engineers - IGTI John P Davis award for exceptional contributions to the literature of gas turbine technology.

Penny Chaloner is a Senior Lecturer in Chemistry at the University of Sussex. She lectures in organic and inorganic chemistry, and occasionally on forensic science and science fiction. Her research interests are in the area of organometallic chemistry, with particular emphasis on catalytic processes using late transition metals that lead to chiral products.

Jo Greenwood is a graduate student in engineering at the University of Sussex. She is working on the development of robust instrumentation for high speed rotating turbomachinery.

Continued on page 95 